\title{
HOW DEADLY IS COVID-19? UNDERSTANDING THE DIFFICULTIES WITH ESTIMATION OF ITS FATALITY RATE
}

\author{
Andrew Atkeson \\ Working Paper 26965 \\ http://www.nber.org/papers/w26965 \\ NATIONAL BUREAU OF ECONOMIC RESEARCH \\ 1050 Massachusetts Avenue \\ Cambridge, MA 02138 \\ April 2020
}

The views expressed herein are those of the author and do not necessarily reflect the views of the National Bureau of Economic Research.

NBER working papers are circulated for discussion and comment purposes. They have not been peer-reviewed or been subject to the review by the NBER Board of Directors that accompanies official NBER publications.

(C) 2020 by Andrew Atkeson. All rights reserved. Short sections of text, not to exceed two paragraphs, may be quoted without explicit permission provided that full credit, including (C) notice, is given to the source. 
How Deadly Is COVID-19? Understanding The Difficulties With Estimation Of Its Fatality

Rate

Andrew Atkeson

NBER Working Paper No. 26965

April 2020

JEL No. A1,C13

\begin{abstract}
$\underline{\text { ABSTRACT }}$
To understand how best to combat COVID-19, we must understand how deadly is the disease. There is a substantial debate in the epidemiological lit- erature as to whether the fatality rate is $1 \%$ or $0.1 \%$ or somewhere in between. In this note, I use an SIR model to examine why it is difficult to estimate the fatality rate from the disease and how long we might have to wait to resolve this question absent a large-scale randomized testing program. I focus on un- certainty over the joint distribution of the fatality rate and the initial number of active cases at the start of the epidemic around January 15, 2020. I show how the model with a high initial number of active cases and a low fatality rate gives the same predictions for the evolution of the number of deaths in the early stages of the pandemic as the same model with a low initial number of active cases and a high fatality rate. The problem of distinguishing these two parameterizations of the model becomes more severe in the presence of effective mitigation measures. As discussed by many, this uncertainty could be resolved now with large-scale randomized testing.
\end{abstract}

Andrew Atkeson

Bunche Hall 9381

Department of Economics

UCLA

Box 951477

Los Angeles, CA 90095-1477

and NBER

andy@atkeson.net

A Matlab Code for paper is available at https://drive.google.com/drive/folders/1kaAFmnbhvnipDEYttDyK3sUvvxqZg21Y?usp=sharing 


\section{Introduction}

There is a large debate in the epidemiology literature regarding the question of how deadly is COVID- $19 .{ }^{1}$ Is the fatality rate $1 \%$ or $0.1 \%$ or somewhere in between? If we envision that $2 / 3$ of all Americans will eventually get the disease absent sustained mitigation measures, then this range of estimates in fatality rates results in a range of cumulative deaths from 2.2 million at the high end and 220,000 at the low end.

What is the nature of this debate? Absent reliable data on the number of active infections and/or the number of people who are now recovered from and resistant to the disease, we are not able to directly compute the fatality rate from COVID-19 from observed deaths. That lack of data leaves us reliant on the use of models to attempt to infer the fatality rate of the disease based on incomplete measurement. Unfortunately, simple epidemiological models are not that helpful in this dimension in the early phase of an epidemic. Absent accurate measurement of disease incidence, one does not learn the true fatality rate until the deaths occur. That observation is the focus on this note.

Specifically, I use a simple SIR model to examine why it is difficult to estimate the fatality rate from the disease and how long we might have to wait to resolve this question absent a large-scale randomized testing program. I focus on uncertainty over the joint distribution of the fatality rate and the initial number of active cases at the start of the epidemic around January 15, 2020. I show how the model with a high initial number of active cases and a low fatality rate gives the same predictions for the evolution of the number of deaths in the early stages of the pandemic as the same model with a low initial number of active cases and a high fatality rate. I then show that the problem of distinguishing these two parameterizations of the model becomes more severe in the presence of effective mitigation measures.

\footnotetext{
${ }^{1}$ See, for example this Op-Ed in the Wall Street Journal from March 24 https: //www.wsj.com/articles/is-the-coronavirus-as-deadly-as-they-say-11585088464 or this news article in Science from March 25 https://www.sciencemag.org/news/2020/03/ mathematics-life-and-death-how-disease-models-shape-national-shutdowns-and-other
} 
The points that I make here regarding the properties of simple SIR models are essentially identical to those made by the epidemiological team at Oxford University in Lourenco et al. $(2020)^{2}$ and are closely related to those in Stock (2020). The presentation of the argument here is intended simply as a pedagogical tool. The model applied to Wuhan in Wang et al. $(2020)^{3}$ is a useful framework for modeling the uncertainties discussed here. Toda $(2020)^{4}$ presents and regularly updates estimates of a SIR model applied to the United States. ${ }^{5}$. Chowell G (2007) is a useful discussion of methods for estimating the transmission rate of an epidemic applied to the 1918-19 Spanish Flu. ${ }^{6}$.

I make no effort to survey the literature on the measurements available of disease incidence. This is a rapidly developing literature. ${ }^{7}$ Hopefully we will obtain sufficient evidence to resolve the debate about the fatality rate of COVID-19 sooner than is implied we might from this model.

In the remainder of this note, I present the model, lay out the measurement assumptions used, and then show model results.

\section{The SIR Model}

The population is set to $N$.

At each moment of time, the population is divided into three categories that sum to the total of $N$. These are susceptible (no immunity) $S$, infected $I$, and resistant

\footnotetext{
${ }^{2}$ available here https://www.medrxiv.org/content/10.1101/2020.03.24.20042291v1

${ }^{3}$ available here https://www.medrxiv.org/content/10.1101/2020.03.03.20030593v1

${ }^{4}$ available here https://arxiv.org/abs/2003.11221

${ }^{5}$ see here for updated estimates https://sites.google.com/site/aatoda111/misc/covid19

${ }^{6}$ available here https://www.ncbi.nlm.nih.gov/pmc/articles/PMC2358966/

${ }^{7}$ See for example this article from March 22 in the South China Morning Post https://www.scmp.com/news/china/society/article/ 3076323/third-coronavirus-cases-may-be-silent-carriers-classified or this commentary by Luigi Zingales from March 17 https://promarket.org/ why-mass-testing-is-crucial-the-us-should-study-the-veneto-model-to-fight-covid-19/
} 
(recovered or dead) $R$.

These fractions of the population evolve over time as follows

$$
\begin{gathered}
d S / d t=-\beta_{t} \frac{S}{N} I \\
d I / d t=\beta_{t} \frac{S}{N} I-\gamma I \\
d R / d t=\gamma I \\
\beta_{t}=R_{t} \gamma
\end{gathered}
$$

The parameter $\gamma$ governs the rate at which agents who are infected become resistant (either recover or die) and hence stop transmitting the disease. The parameter $\beta_{t}$ is the rate at which infected agents spread the virus to others that they encounter. This parameter is a reduced form parameter that is impacted by the biological disease transmission mechanism, the rate at which agents bump into each other, and the extent to which agents use prophylactics in their meetings. This parameter can thus be impacted by mitigation measures such as social distancing and the use of masks, etc.

The parameter $R_{t}=\beta_{t} / \gamma$ is the normalized transmission rate. This parameter corresponds to the parameter cited in many news and academic studies. Note that we can restate the equations of the model in terms of this parameter as

$$
\begin{gathered}
d S / d t=-R_{t} \gamma \frac{S}{N} \\
d I / d t=\left(R_{t} \frac{S}{N}-1\right) \gamma I \\
d R / d t=\gamma I
\end{gathered}
$$

We denote the fatality rate from the disease by $\nu$. That is, $\nu$ is the fraction of agents who are resistant who are so because they died. Thus, cumulative deaths are 
given by $D=\nu R$. The death rate per unit time is given by

$$
d D / d t=\nu d R / d t
$$

The initial conditions of the model are $R=0, I=I_{0}$ and $S=N-I_{0}$ at date 0 .

Note that in this model I abstract from the distinction between the time it takes to stop being infections $(\gamma)$ and the time it takes to die from the disease. As discussed in the Oxford study, this distinction can be important for the quantitative implications of the model.

\subsection{Model Properties:}

The following properties of the model are standard.

1. Model steady-states have $I=0$. If $I=0$, then any combination of $S$ and $R$ that sum to $N$ is a steady-state.

2. If $I>0$, then $d I / d t<=0$ if and only if $R_{t} \frac{S}{N} \leq 1$. Thus, the steady-states reached from an initial value of $I>0$ must have $S / N \leq 1 / R_{t}$.

3. The growth rate of the log of the number of active cases is given by

$$
\frac{d \log I}{d t}=\left(R_{t} \frac{S}{N}-1\right) \gamma
$$

4. The growth rate of the log of the total number of cases is given by

$$
\frac{d \log (I+R)}{d t}=R_{t} \gamma \frac{S}{N} \frac{I}{1-S}
$$

5. Under the assumption of a constant mortality rate, the growth rate of the log of the cumulative number of deaths is equal to the growth rate of the log of 
the cumulative number of recovered cases and is given by

$$
\frac{d \log D}{d t}=\frac{d \log R}{d t}=\gamma \frac{I}{R}
$$

When $R_{t}$ is constant, an analytical solution of the model is available. ${ }^{8}$

\section{Measurement:}

Assume that we have data on deaths $D$. That is, assume that we can measure both the level and the growth rate of $D$. Note that this is a controversial assumption, ${ }^{9}$ but we make this assumption here to illustrate the problem of estimating $\nu$ in this simple model in a stark manner.

Assume that we have a plausible range of estimates of $\gamma$, denoted by $\tilde{\gamma} \in\left[\gamma_{\text {min }}, \gamma_{\text {max }}\right]$, taken from clinical measurements of the progress of the disease. (Much of the uncertainty here is about how soon cases become infectious and whether there are unmeasured cases that have different properties of disease progression). In the illustrations that I consider, I will set $\gamma=1 / 7$.

Consider now measurement of the number of resistant individuals $R$. Clearly, if we had accurate measures of the number of cases recovered $R$ and the number of deaths due to the disease $D$, it would be straightforward to estimate the fatality rate $\nu=D / R$. Hence, given accurate data on deaths, a large scale testing program that would allow us to identify those who have had the disease and an accurate count of those who died from the disease would allow for direct estimation of the fatality rate $\nu$. (See, for example, the plan in Germany to test 100,000 people at

\footnotetext{
${ }^{8}$ See https://advanceseng.com/exact-analytical-solutions-susceptible-infected-recovered-sir-epidemic-model-sir-mod ${ }^{9}$ See, for example concerns about the measurement of deaths due to COVID-19 in Italy here https://www.corriere.it/politica/20_marzo_26/ the-real-death-toll-for-covid-19-is-at-least-4-times- the-official-numbers-b5af0edc-6eeb-11ea-925b-a0c3cdbe1130. shtml
} 
random for antibodies to the disease. ${ }^{10}$ ) For the remainder of this note, I assume that independent measures of $R$ are not available. That is, I assume that measures of $R$ are derived by tracking confirmed active cases as these cases are resolved as is done on this website. ${ }^{11}$

Consider next measurement of the number of active infections $I$. Note that if one had accurate measures of the number of people actively infected, $I$, then one could use data on the growth rate of the log of deaths and an estimate of the parameter $\gamma$ to infer the number of resistant individuals $R$ using equation 6 . I assume that an accurate count of the number of actively infected individuals is not available. Instead, I assume that we have data on active infections up to a constant, proportional measurement error. That is, assume that we observe $\tilde{I}=\eta_{I} I$ where the proportional measurement error $\eta_{I}$ is unknown. This assumption that we observe active cases up to a constant, proportional, measurement error is likely an overstatement of the data available as testing policies have been constantly changing. Again, we make this assumption here to illustrate the problem of estimating $\nu$ in this simple model in a stark manner.

Consider now the measurement of $R_{t}$. Under the assumption that we observe the number of active infections up to proportional measurement error, we can measure the growth of the log of infections but not the level of infections. Note then from equation 4 , we can estimate $R_{t}$ in the earliest phase of the epidemic when we know that $S / N$ is close to 1 if we have an estimate of the parameter $\gamma$ from clinical data. For the purposes of the illustrations here, we assume that $R_{t}$ in the earliest phase of the epidemic without mitigation is equal to 2.5 .

I now consider the difficulty of measuring the fatality rate $\nu$ in the early phase of the epidemic given reasonable uncertainty over the initial condition $I_{0}$.

Assume that we have a plausible range of initial cases introduced into the United

\footnotetext{
${ }^{10}$ https://www.dailymail.co.uk/news/article-8170903/Germany-100-000-people-coronavirus-antibody-tests. html

${ }^{11}$ See, for example, the statistics at https://www.worldometers.info/coronavirus/
} 
States due to people returning from abroad with infections $\tilde{I}_{0} \in\left[I_{0, \min }, I_{0, \max }\right]$. Start the model on January 15. In the illustrations that I consider, I will set $I_{0}$ equal to either 330 active cases on January 15 or, alternatively 3300 active cases on January 15. This uncertainty would correspond to difficulties of measuring infections among returning travelers and initial bursts of infection occurring in super-spreading events.

I now solve the model to demonstrate that the model's implications for cumulative deaths in the early phase of the epidemic are very similar under the assumption that $I_{0}=330$ and the fatality rate $\nu=0.01(1 \%)$ or the alternative assumption that $I_{0}=3300$ and the fatality rate $\nu=0.001(1 / 10$ of $1 \%)$ despite the fact that these two alternative parameter assumptions have an order of magnitude difference in their implications for cumulative deaths over the long term.

I refer to the parameter combination of $I_{0}=330$ and $\nu=0.01$ as the high fatality case and the combination of $I_{0}=3300$ and the fatality rate $\nu=0.001$ as the low fatality case.

I consider two computational experiments in the next two sections. In the first, I compute the implications of the model for deaths and active infections under the high and low fatality cases with the normalized transmission rate $R_{t}$ held constant at 2.5. I then consider the implications of the model under the high and low fatality cases under the assumption that the normalized transmission rate $R_{t}$ drops rapidly from 2.5 to 1.25 , corresponding to a $50 \%$ reduction in transmission due to mitigation.

\section{Model Experiment 1: Constant $R_{t}$}

Let us now look at the model implications for the evolution of $I, R$, and deaths $D$ under the assumption that $I_{0}=330$ and the fatality rate $\nu=0.01(1 \%)$ and the alternative assumption that $I_{0}=3300$ and the fatality rate $\nu=0.001(1 / 10$ of $1 \%)$ when we set $R_{t}=2.5$ for all $t$.

In Figure 1, I show the path for the cumulative number of deaths in the high and 
low fatality cases with $R_{t}$ constant at 2.5. We see clearly in this figure that these two alternative parameter configurations have dramatically different implications for the long-run number of deaths - something close to 3 million in one case and 300,000 in the other.

In Figure 2, I zoom in on the implications of the model for the first 180 days. I plot the log of the cumulative number of deaths in the high and low fatality cases with $R_{t}$ constant at 2.5. I omit data from the first 20 days as the initial number of deaths is so low that taking logs is problematic. We see in this figure that the implications of the model for deaths in the high and low fatality cases are virtually impossible to distinguish in the first 45-50 days. After 60 days, the non-linearities in the model begin to kick in to allow the two cases to be distinguished. (Note that in this initial phases of the epidemic between days 20 and 40, the doubling time of deaths with these parameters is on the order of 3.3 days regardless of the death rate). 


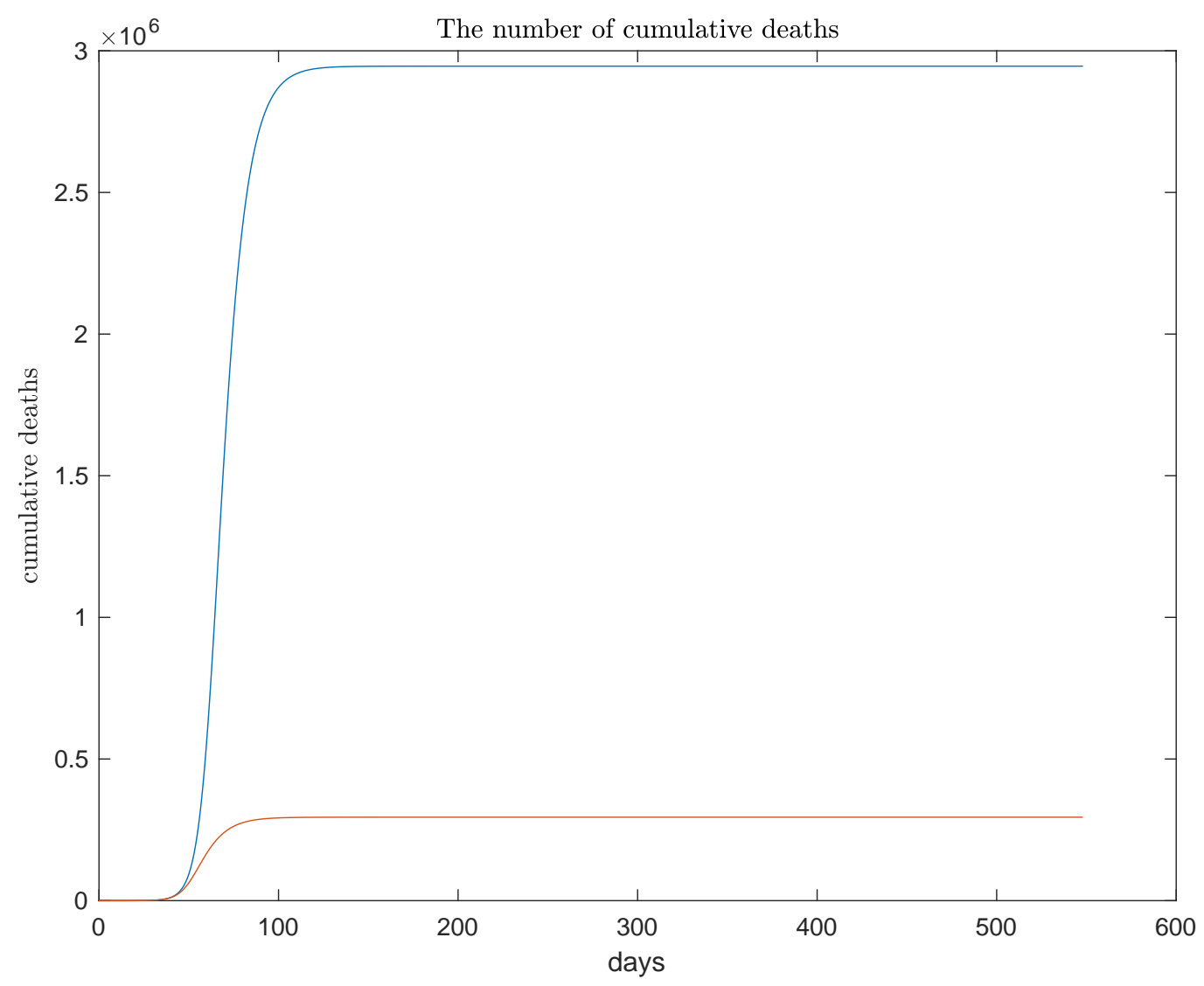

Figure 1: Cumulative Deaths over 18 months with $\nu=1 \%$ and $\nu=0.1 \%$ 


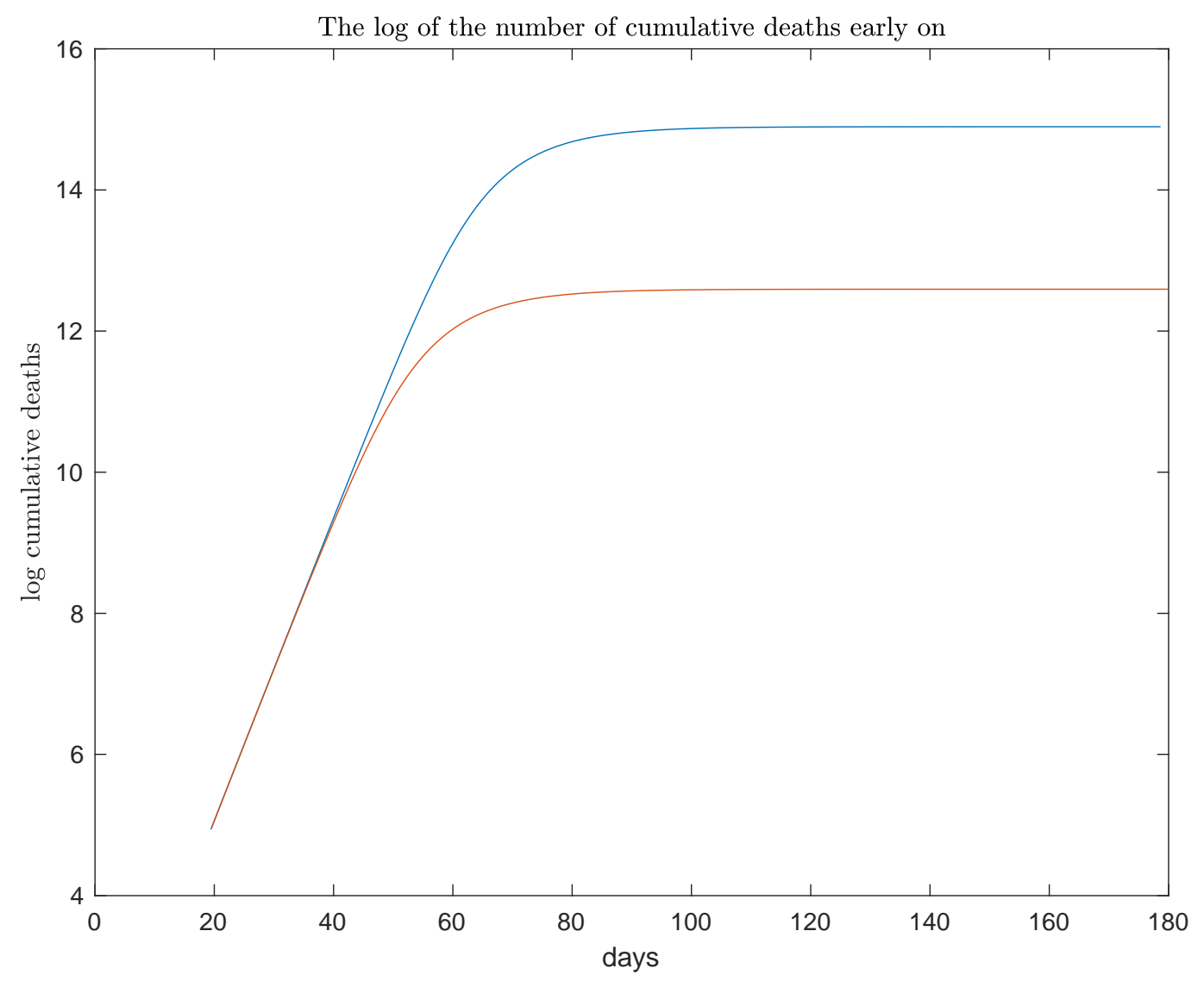

Figure 2: $\log$ of Cumulative Deaths over 120 days with $\nu=1 \%$ and $\nu=0.1 \%$

How is it that the model gives such similar implications for deaths in the early phase of the epidemic under these dramatically different assumptions about the fatality rate of the disease? The answer has to do with the differences in the model's implications for the number of active cases $I$ and resistant population $R$.

In figure 3, I show the cumulative number of cases and the number of actively infected under the high and low fatality scenario. Because, under the high fatality scenario, the initial condition for $I_{0}$ is higher, cumulative cases and active infections in that case lead those in the low fatality scenario. Around day 45 of the epidemic, 
the differences in the model implications for $R+I$ and $I$ under these two alternative parameter configurations is enormous. After day 100, this difference has completely disappeared. As a result, the fatality rate $\nu$ becomes obvious after that date simply because the deaths do not materialize in the low fatality case.

In Figure 4, I show the logarithm of active infections $I$ and cumulative cases $R+I$ under the two parameter configurations considered. We see in this figure that the ratio of $I$ to $I+R$ behaves very similarly across the two parameter configurations during the period from 20 to 45 days. We see clearly that the series across the two parameter configurations are simply proportional shifts of each other. Thus, data on active infections and cumulative cases would not be helpful in distinguishing the two cases if we do not have information about the proportional measurement error of active infections $\eta_{I}$.

To gain rough intuition for the results here, consider the evolution of the model under the simplifying assumption of no congestion in the transmission of the disease. That is, consider an approximation to our model in the early phase of the epidemic in which $S / N$ is close to one. Then we have

$$
\begin{gathered}
d I / d t \approx\left(R_{t}-1\right) \gamma I \\
d R / d t=\gamma I
\end{gathered}
$$

As these equations make clear, the level of $R$ at any date in the initial phase of the epidemic simply scales in the initial condition $I_{0}$ and thus the model implications for the fatality rate

$$
\tilde{\nu}=D / R
$$

varies inversely with the initial condition $I_{0}$. It becomes possible to distinguish the high and low fatality cases only when the non-linearities induced by the congestion term $S / N$ kick in. 


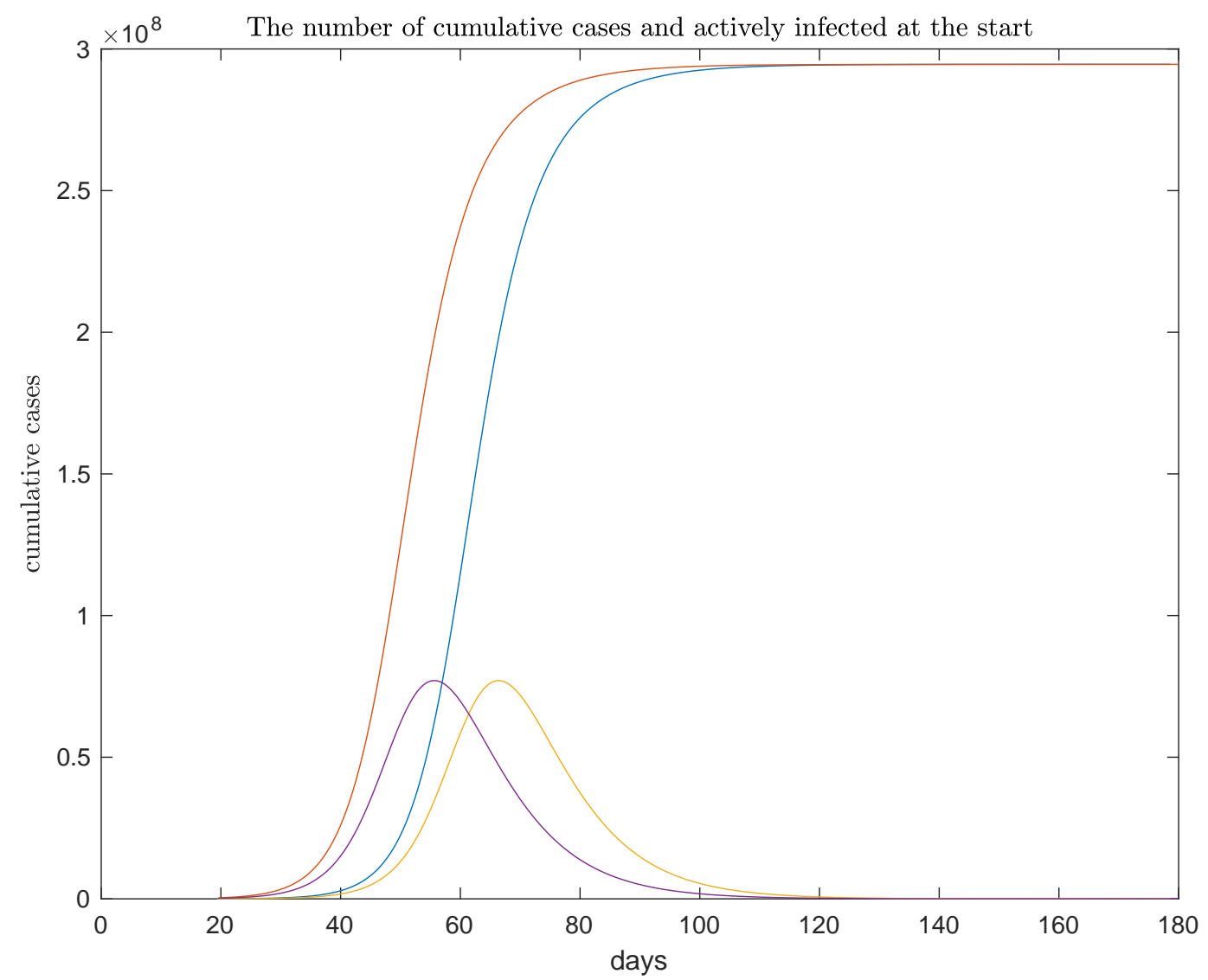

Figure 3: Cumulative cases and active infections under the high and low fatality cases 


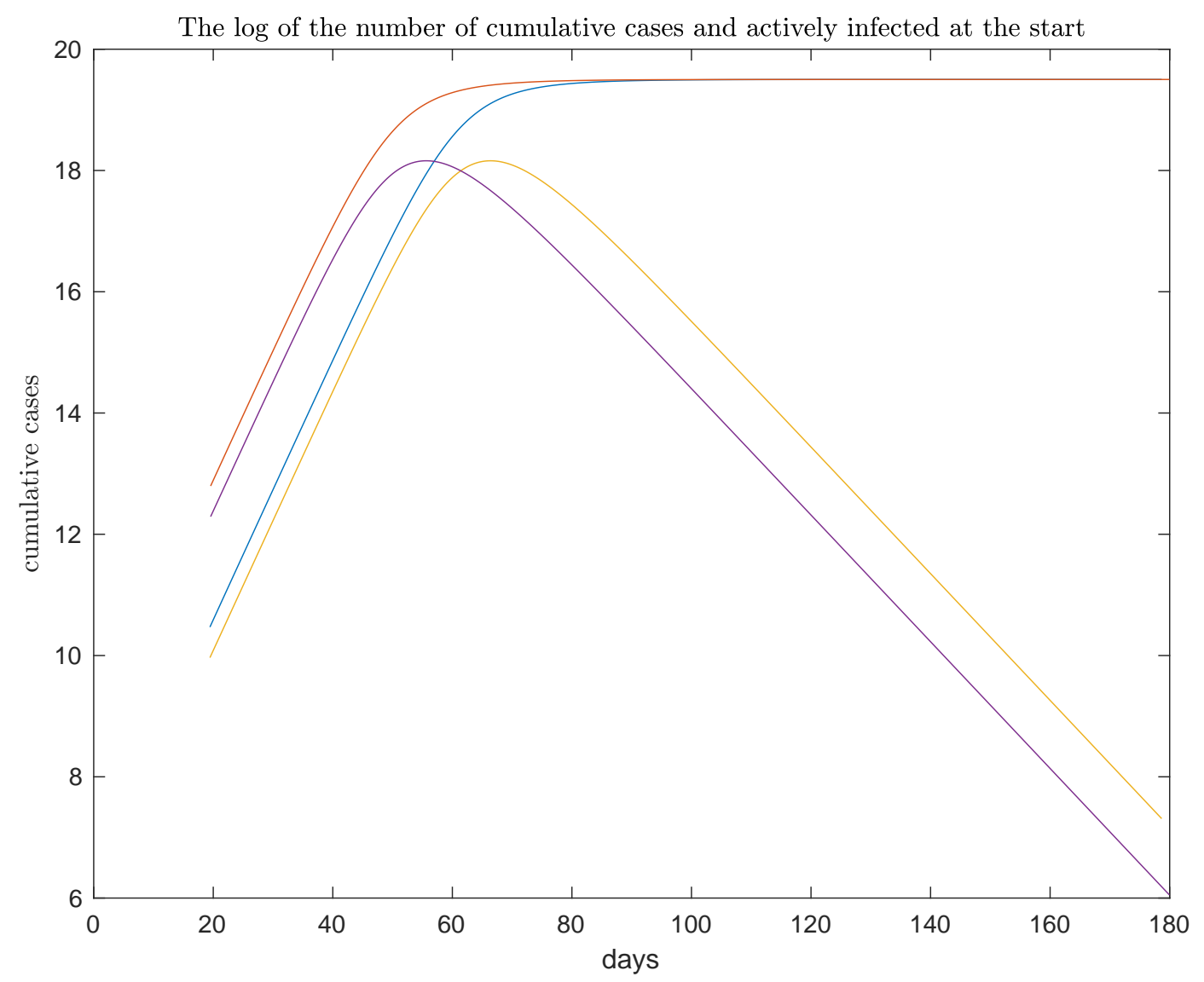

Figure 4: The log of cumulative cases and active infections under the high and low fatality cases

\section{$5 \quad$ Model Experiment 2: Falling $R_{t}$}

Let us now look at the model implications for the evolution of $I, R$, and deaths $D$ under the assumption that $I_{0}=330$ and the fatality rate $\nu=0.01(1 \%)$ and the alternative assumption that $I_{0}=3300$ and the fatality rate $\nu=0.001(1 / 10$ of $1 \%)$ when we set $R_{t}=2.5$ to start and then have it fall rapidly to $R_{t}=1.25$ due to long-lasting mitigation measures. Again, we refer to the parameter combination of $I_{0}=330$ and $\nu=0.01$ as the high fatality case and the combination of $I_{0}=3300$ 
and the fatality rate $\nu=0.001$ as the low fatality case.

In Figure 5, I show the path for the cumulative number of deaths in the high and low fatality cases with $R_{t}$ starting at 2.5 and then falling permanently to 1.25 due to long-term mitigation measures. We see clearly in this figure that mitigation lowers the cumulative deaths under these two alternative parameter configurations, but these two configurations still have dramatically different implications for the long-run number of deaths - something close to 1.2 million in one case and 120,000 in the other.

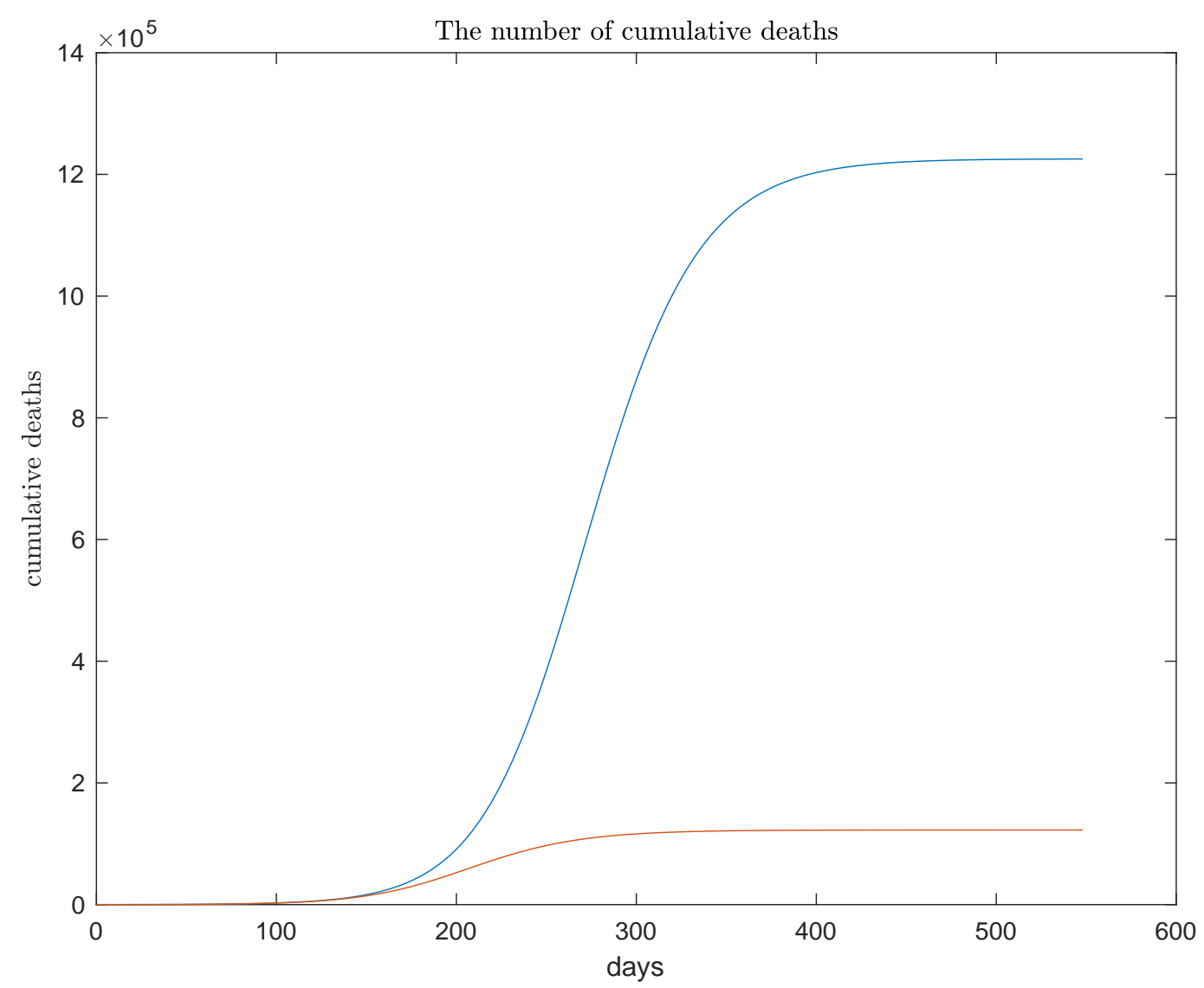

Figure 5: Cumulative Deaths over 18 months with $\nu=1 \%$ and $\nu=0.1 \%$ 
In Figure 6, I zoom in on the implications of the model for deaths in the first 180 days. I plot the log of the cumulative number of deaths in the high and low fatality cases with $R_{t}$ starting at 2.5 and then falling rapidly to 1.25 and remaining at that level permanently. I omit data from the first 20 days as the initial number of deaths is so low that taking logs is problematic. We see in this figure that the implications of the model for deaths in the high and low fatality scenarios are virtually impossible to distinguish in the first 120 days. Clearly, aggressive mitigation makes the problem of estimating the fatality rate of the disease more severe.

The rough intuition for this result is that with a lower transmission rate, it takes longer for the model non-linearities due to congestion in transmission $(S / N)$ to kick in. 




Figure 6: $\log$ of Cumulative Deaths over 120 days with $\nu=1 \%$ and $\nu=0.1 \%$

\section{Conclusion:}

This note serves only as an illustration of the difficulties of measuring the fatality rate COVID-19 (or any other disease) in the early phases of the epidemic absent accurate measurement of either resolved cases $R$ or active infections $I$. The obvious policy conclusion, made by many others, is that widespread testing is needed to understand this disease. Absent that testing, we may simply have to wait to see whether lots of people die to get an accurate estimate of the danger of this disease. Let us hope 
that the data from the German testing program becomes available soon. 


\section{References}

Bettencourt LM. Chowell G, Nishiura H. Comparative estimation of the reproduction number for pandemic influenza from daily case notification data. $J R$ Soc Interface, 4(2):155-166, Feb 222007.

Jose Lourenco, Robert Paton, Mahan Ghafari, Moritz Kraemer, Craig Thompson, Peter Simmonds, Paul Klenerman, and Sunetra Gupta. Fundamental principles of epidemic spread highlight the immediate need for large-scale serological surveys to assess the stage of the sars-cov-2 epidemic. Cold Spring Harbor Laboratory Press, March 262020.

James H. Stock. Data gaps and the policy response to the novel coronavirus. March 252020.

Alexis Akira Toda. Susceptible-infected-recovered (sir) dynamics of covid-19 and economic impact. Cornell University, March 252020.

Chaolong Wang, Li Liu, Xingjie Hao, Huan Guo, Qi Wang, Jiao Huang, Na He, Hongjie Yu, Xihong Lin, An Pan, Sheng Wei, and Tangchun Wu. Evolving epidemiology and impact of non-pharmaceutical interventions on the outbreak of coronavirus disease 2019 in wuhan, china. Cold Spring Harbor Laboratory Press, March 62020. 